\title{
Paediatric Asthma:European Respiratory Monograph Number 56:Kai-Hakon and Jorrit Gerritsen (eds)
}

\author{
Published by European Respiratory Society (ERS), June 2012, Paperback binding, Pages: \\ 233, Price: US\$ 80
}

\author{
S. K. Kabra
}

Received: 3 August 2012 /Accepted: 7 August 2012 /Published online: 30 August 2012

(C) Dr. K C Chaudhuri Foundation 2012

Asthma in children is one of the leading causes of emergency room visit, absence from the school, economic and social impact on the family, society and country. Research over the last two decades have helped in understanding pathophysiology and improving the management. Physicians treating asthma need to keep themselves updated on various aspects of asthma in children.

This official monograph of ERS on Pediatric Asthma, as part of series of clinical handbook for respiratory physicians presents updated information on various aspects of asthma in children in detail. It includes 19 well researched articles written by experts in the field of asthma.

Monograph covers different aspects of asthma including various phenotypes in relation to age, role of infections and allergens, genetics and epidemiology, role of indoor and outdoor environmental pollution, feasibility and reproducibility of documentation of airway hyper responsiveness in different age groups, treatment of asthma in different age groups and treatment of severe problematic asthma. It also includes an article on economic and familial impact and effect of psychological factors. In addition, it also includes chapters on follow up and some details of new and future development in pediatric asthma.

Each chapter includes an abstract that gives summary of the contents of article followed by extensive evidence based review and discussion of the subject and at the end, a conclusion of the findings and final recommendations. In almost all the articles, authors have highlighted gaps in knowledge and challenges that needs to be answered by appropriate research. Authors have used various modalities such as tables, graphs and algorithms to explain the subject. A detailed description and controversies in pediatric asthma including diagnostic dilemma, uncertainties about pharmacotherapy, especially in preschool wheezing, issues related to pharmacotherapy in different age groups are discussed in different chapters.

This monograph will enhance understanding of asthma and help in better management of asthma in children in all the age groups. It is very useful reading for Pediatric pulmonologists, post graduate students, researchers and practioners managing asthmatic children.

S. K. Kabra $(\bowtie)$

Pediatric Pulmonology Division, Department of Pediatrics,

All India Institute of Medical Sciences,

New Delhi 110029, India

e-mail: skkabra@live.in 